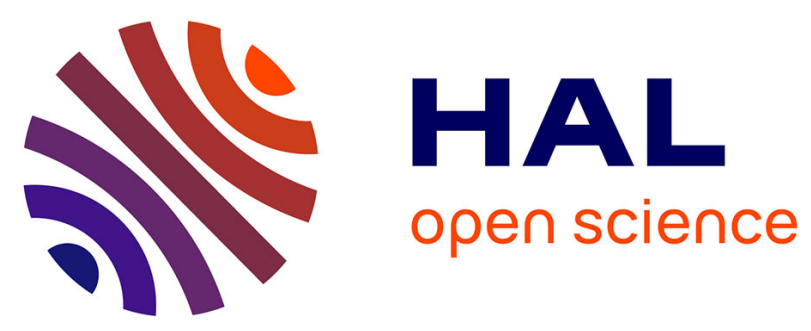

\title{
Orbital Angular Momentum Transfer to Stably Trapped Elastic Particles in Acoustical Vortex Beams
}

Diego Baresch, Jean-Louis Thomas, Régis Marchiano

\section{To cite this version:}

Diego Baresch, Jean-Louis Thomas, Régis Marchiano. Orbital Angular Momentum Transfer to Stably Trapped Elastic Particles in Acoustical Vortex Beams. Physical Review Letters, 2018, 121 (7), pp.074301. 10.1103/PhysRevLett.121.074301 . hal-02332830v2

\section{HAL Id: hal-02332830 \\ https://hal.science/hal-02332830v2}

Submitted on 25 Oct 2019

HAL is a multi-disciplinary open access archive for the deposit and dissemination of scientific research documents, whether they are published or not. The documents may come from teaching and research institutions in France or abroad, or from public or private research centers.
L'archive ouverte pluridisciplinaire HAL, est destinée au dépôt et à la diffusion de documents scientifiques de niveau recherche, publiés ou non, émanant des établissements d'enseignement et de recherche français ou étrangers, des laboratoires publics ou privés. 


\title{
Orbital Angular Momentum Transfer to Stably Trapped Elastic Particles in Acoustical Vortex Beams
}

\author{
Diego Baresch* and Jean-Louis Thomas \\ Sorbonne Université, CNRS UMR 7588, Institut des NanoSciences de Paris, INSP, F-75005 Paris, France \\ Régis Marchiano \\ Sorbonne Université, CNRS UMR 7190, Institut Jean le Rond d'Alembert, F-75005 Paris, France
}

(Received 6 April 2018; published 14 August 2018)

\begin{abstract}
The controlled rotation of solid particles trapped in a liquid by an ultrasonic vortex beam is observed. Single polystyrene beads, or clusters, can be trapped against gravity while simultaneously rotated. The induced rotation of a single particle is compared to a torque balance model accounting for the acoustic response of the particle. The measured torque $\left(\sim 10 \mathrm{pN} \mathrm{m}\right.$ for a driving acoustic power $\left.\sim 40 \mathrm{~W} / \mathrm{cm}^{2}\right)$ suggests two dominating dissipation mechanisms of the acoustic orbital angular momentum responsible for the observed rotation. The first takes place in the bulk of the absorbing particle, while the second arises as dissipation in the viscous boundary layer in the surrounding fluid. Importantly, the dissipation processes affect both the dipolar and quadrupolar particle vibration modes suggesting that the restriction to the well-known Rayleigh scattering regime is invalid to model the total torque even for spheres much smaller than the sound wavelength. The findings show that a precise knowledge of the probe elastic absorption properties is crucial to perform rheological measurements with maneuverable trapped spheres in viscous liquids. Further results suggest that the external rotational steady flow must be included in the balance and can play an important role in other liquids.
\end{abstract}

DOI: 10.1103/PhysRevLett.121.074301

Since the demonstration of particle trapping and manipulation of transparent particles by a single focused laser beam by Ashkin et al. [1], "optical tweezers" that can pull a trapped particle in all three directions have found numerous applications, particularly in the biophysical research $[2,3]$. Using the radiation pressure of sound, rather than light, it was recently demonstrated that "acoustical tweezers" could operate as three-dimensional traps for elastic particles using a single ultrasonic vortex beam first numerically [4] and then experimentally $[5,6]$. The change in nature of the propagating wave presents several advantages for contactless manipulation such as the possibility to operate through turbid media, allow penetration in tissue, and largely increase the magnitude of the trapping force and the size of the particles.

The attraction in the direction of the intensity gradient of transparent (high optical index) dielectric objects in optical tweezers relies on the transfer of the momentum carried by photons. It is, however, well established that photons can also carry angular momentum and exert torques [7]. This important degree of freedom has proven important for the controlled rotation of optically trapped particles with the spin or orbital angular momentum (OAM) of photons [8-12].

In contrast, longitudinal acoustic waves in liquids do not carry momentum [13], but instead can induce a mean stress, after exchange of a flux of momentum-e.g., by scattering or absorption-either it be linear or angular. Rayleigh first analyzed and quantified the torque exerted on a disk suspended in a sound field [14]. In that particular case the torque is understood as a consequence of the uneven radiation pressure exerted on the surface of a disk unaligned with the sound propagation direction [15] in a way that any object of irregular form could experience a net radiation torque. Accounting for the finite size of the viscous boundary layer $\delta=\sqrt{(2 \mu / \rho \omega)}$ around an object's surface, where $\omega$ is the pulsation, $\mu$ the dynamic viscosity, and $\rho$ the density of the suspending fluid, respectively, the elliptical motion of fluid particles in a system of out-ofphase orthogonal standing waves can induce the rotation of axisymmetric objects $[16,17]$. Combined with acoustical levitation $[18,19]$, systems of counterpropagating waves have been selected as an advantageous method to induce the rotation of matter in air $[20,21]$ and are at the basis of the rotation of spherical, cylindrical, and anisotropic particles, including cells, in fluids [22-25].

The momentum flux vector, or Poynting vector, of an acoustical vortex (AV) will locally point in the direction of the helicoidal wave front offering an additional degree of freedom under which it will be exchanged: the OAM of sound. The direct OAM transfer to matter has been observed in air [26,27] and water [28-30] through absorption or chiral scattering [31]. AVs have recently been used to simultaneously levitate and rotate particles in air [32] but the lack of viscosity leads, however, to an off-axis 
rotational instability that can be controlled at the expense of the decrease of the net OAM transfer [33]. Nonetheless, the physical mechanisms driving the acoustic torque are unclear. No absorption processes were considered, suggesting that the main mechanisms leading the particle to spin around its axis were overlooked. Additionally, the demonstration of the coexistence of the axial negative gradient force [6] and driving torque is not evident. A negative gradient force, pulling a particle against the acoustical momentum flux, is a crucial feature in the development of acoustical tweezers. In liquids, acoustic radiation forces and torques have experimentally been shown to exceed by 6 orders of magnitude their optical counterpart [28,29,34]. Hence, despite the significant potential of combining simultaneous trapping and rotation with a single beam for selective particle manipulation, a quantitative experimental test of acoustical OAM transfer models is still missing.

In this Letter, we report on the observation of the simultaneous rotation and trapping of particles pulled by a negative gradient force. This is done by using AV-based acoustical tweezers to stably trap janus polystyrene particles (see Fig. 1) and measuring their rotation rate. The steady spinning frequency is used to derive a torque balance based on analytical calculations of the nonlinear acoustical torque. We find that, for a single spherical polystyrene bead, it is crucial to fully model the elastic scattering beyond the usual long-wavelength regime that is found to be invalid for the acoustic torque, within the usual size bonds set for this limit. For a particle size of $a / \lambda \sim 0.13$ where $\lambda$ is the driving wavelength, the dissipation in the solid particle bulk and viscous boundary layers involved in the dipolar and quadrupolar oscillations is responsible for

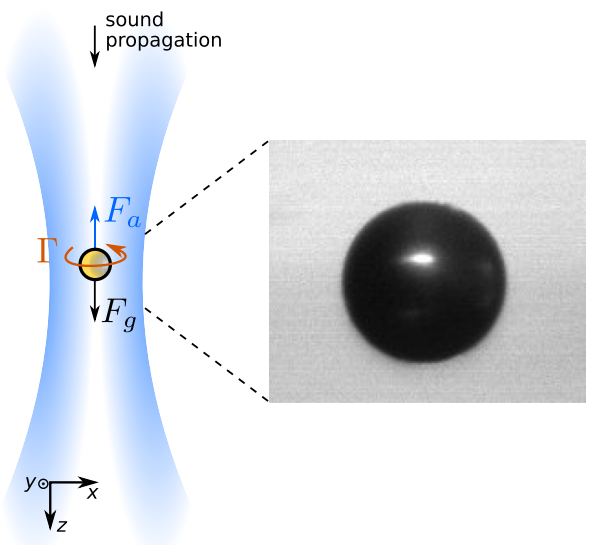

FIG. 1. Schematic view of the ultrasonic vortex beam trapping a janus polystyrene particle coated with a thin layer of gold. The negative acoustic gradient force, $F_{a}$, balances the gravity force $F_{g}$. The transfer of OAM from the beam to the particle and fluid bulk applies a torque $\Gamma$ driving an on-axis rotation. A photograph of a trapped and rotating particle is also shown. the OAM transfer and driving torque. Further observations demonstrate the stable trapping and controlled rotation of asymmetric clusters of particles.

Experimental setup.-Figure 1 shows a schematic of the setup and a photograph of a trapped and spinning particle. Using the experimental setup previously described [6], a focused $\mathrm{AV}$ (frequency $f=1.15 \mathrm{MHz}$, wavelength $\lambda=$ $c_{0} / f=1.3 \mathrm{~mm}$ for a speed of sound $c_{0}=1480 \mathrm{~m} / \mathrm{s}$ at $20^{\circ} \mathrm{C}$ ) exerts a lateral force trapping the particle in its zero pressure core and the tight focusing gives rise to the negative pulling force operating against gravity (Movie 1 in the Supplemental Material [35]). Polystyrene beads (Polysciences Inc., of radius $2 a \sim 360 \mu \mathrm{m}$ ) were partially coated with a nanometric layer of gold to optically observe their rotational motion at a rate of 159 frames per second. The acoustic properties of the particles are unaltered by the coating procedure. The OAM of an AV with topological charge $m$ (phase variation $e^{i m \varphi}$ around the $z$ axis) is related to the total energy density $E$ by $M=(m / \omega)\langle E\rangle$, [48]. It is thus possible to increase the torque by increasing the value of $m$. An immediate consequence is, however, the reduction of the linear momentum. We have previously analyzed theoretically that this translates into the reduction of the stiffness of the acoustic trap in both axial and lateral directions [4]. Hence, for the purpose of demonstrating simultaneous pulling against gravity and spinning in a context where gravity plays an important role, vortex beams with the minimal topological charge $m= \pm 1$ are generated. The maximum acoustic pressure used is $p_{0}=$ $0.8 \pm 0.1 \mathrm{MPa}$ measured on the vortex ring or equivalently an intensity of $42 \mathrm{~W} / \mathrm{cm}^{2}$. Note that higher order AVs with $m=3$ and 5 were recently used in air to levitate expanded polystyrene particles [33]. However, only the acoustic pushing (positive) force was observed to counteract the pull of gravity, the acoustic negative gradient force being insufficient as predicted theoretically [4]. Thus, the current failure of using high order AVs in single-beam tweezers is so far common to both air and water.

OAM transfer balance.--Upon starting the AV emission, two different polystyrene particles of, respectively, $a=$ $172 \pm 4 \mu \mathrm{m}$ and $174 \pm 4 \mu \mathrm{m}$ radius are accelerated towards their equilibrium position where all forces balance out. The OAM transfer of a vortex beam of topological charge $m=-1$ results in the rotation at a rate of $f_{r}=10.5$ and $11 \mathrm{~Hz}$, respectively [Fig. 2(a)]. The spinning rate is detected by the optical mean gray value extracted from each frame of a video and displayed (Methods in the Supplemental Material [35]). The dark side of the particle appears twice in a revolution when the golden face lies in between the light source and the camera (see Movie 1 in the Supplemental Material [35]). The angular speed $\Omega=$ $2 \pi \times f_{r} \simeq 70 \mathrm{rad} / \mathrm{s}$ suggests that the driving acoustic torque $\Gamma$ on the spheres is balanced by the drag $\Gamma_{D}=-8 \pi \mu a^{3} \Omega \simeq$ $-10 \mathrm{pN} \mathrm{m}$ acting in the opposite direction in a fluid of density $\rho=1000 \mathrm{~kg} / \mathrm{m}^{3}$ and viscosity $\mu=1 \mathrm{mPas}$ for 
which the low Reynolds number approximation $\mathrm{Re}=$ $a^{2} \Omega / \nu \ll 1$ holds. Note that inertial effects acting on a time scale of a few milliseconds [23] are unresolved with this setup.

Among the possible means to induce an OAM transfer, Anhäuser et al. invoked the rotational flow induced in the bulk of a viscous mixture of aqueous glycerol by the absorption of an incident AV [29]. Though water has a much lower viscosity, here we were able to directly observe the flow by injecting a solution of ink for which the diffusion time scale was much shorter than for the acoustically forced flow. In Fig 2(b), we can follow the temporal evolution of the flow in the focal zone by looking at the evolution of the ink concentration (Movie 2 in the

(a)

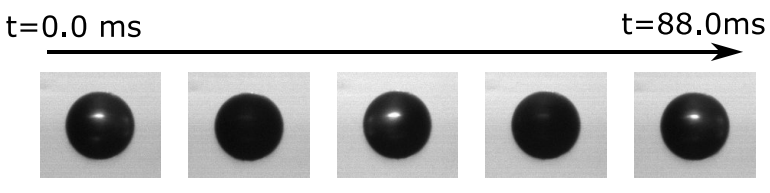

(b)

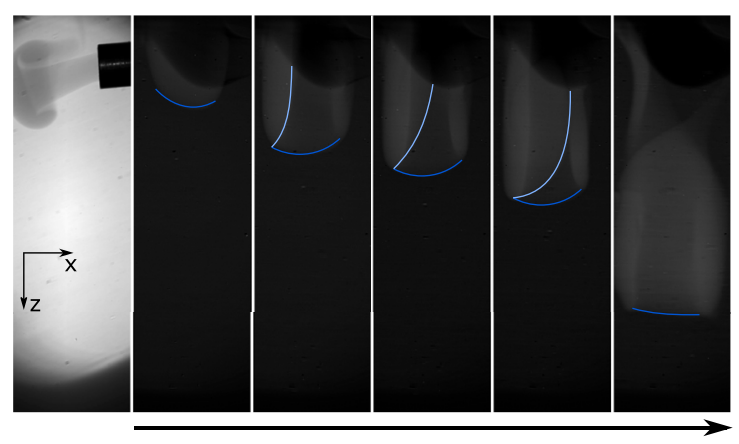

$\mathrm{t}=0.0 \mathrm{~s}$ $\mathrm{t}=1.5 \mathrm{~s}$

(c)

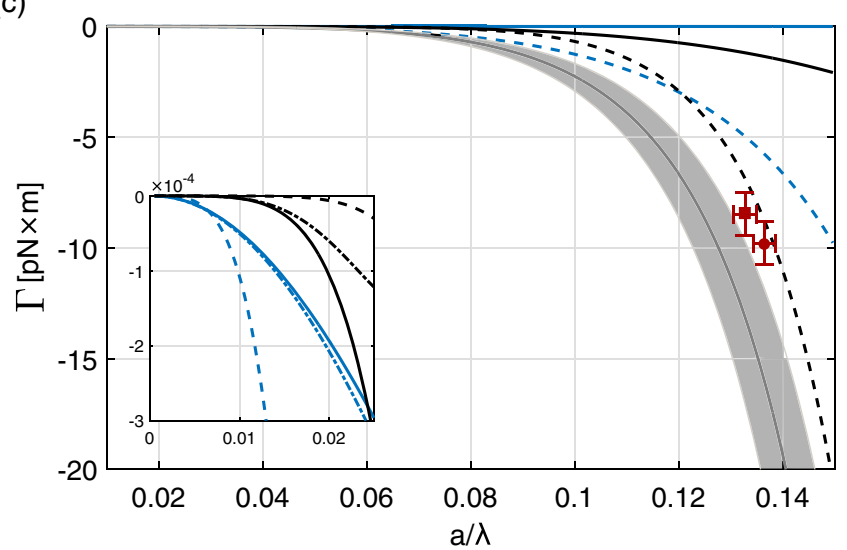

FIG. 2. OAM transfer to a stably trapped particle. In (a) snap shots are shown of one complete revolution of a polystyrene particle with $a=174 \mu \mathrm{m}$. (b) Rotational acoustic flow generated by the absorption of the AV in the fluid bulk. An injected drop of ink allows for the observation of the developing flow upon emission of the AV. (c) Computation of the acoustic torque as a function of the particle radius and particle vibration modes (see text). The measured torque with error bars (see text) are added for two independent rotation experiments with spherical probes having $a=172$ and $174 \mu \mathrm{m}$, respectively.
Supplemental Material [35]). It reveals the simultaneous axial and rotational components of the flow field that exists without the presence of the sphere. The rotational flow can be evaluated (see the Supplemental Material [35]) to be approximately $u_{\varphi} \sim 1 \mathrm{~mm} / \mathrm{s}$ in the vicinity of the trapped sphere, weaker than the axial flow, $u_{z} \sim 4 \mathrm{~mm} / \mathrm{s}$. While devising a method to measure the fine features of this complex 3D flow was beyond the scope of this study, we infer that it contributes to weakly reduce the drag on the sphere, introducing an approximate error on the torque $\Gamma_{D}^{\prime}=8 \pi \mu a^{3}\left(u_{\varphi} / a\right) \simeq 1 \mathrm{pN} \mathrm{m}$.

The mechanical torque driving the rotation is the timeaveraged acoustic torque exerted on the particle partly scattering and absorbing the incident OAM. As described in the Supplemental Material [35], we compute the torque $\Gamma$ exerted by the focused $\mathrm{AV}$ on a polystyrene bead in water with density $\rho_{p}=1050 \mathrm{~kg} / \mathrm{m}^{3}$ and speed of sound $c_{\ell}^{\infty}=$ $2350 \mathrm{~m} / \mathrm{s}$ and $c_{t}^{\infty}=1100 \mathrm{~m} / \mathrm{s}$ for longitudinal and transverse internal waves, respectively. Polystyrene, as other amorphous solids with a glass transition, has a viscoelastic behavior well modeled as a Maxwell material. Thus the bulk and shear absorption coefficients are frequency dependent and ultrasonic measurements have been reported in the literature [36]. The longitudinal absorption coefficient $\alpha_{\ell}=30 \mathrm{~Np} / \mathrm{m}$ is found to be roughly 3 times weaker than its transverse counterpart $\alpha_{t}=100 \mathrm{~Np} / \mathrm{m}$. We also account for losses involved in the boundary layer supporting shear viscosity waves $[37,38]$. This dissipation will also give rise to a steady mean flow, originally analyzed by Schlichting [39]. It's influence in calculating the torque is discussed in the Supplemental Material [35]. The field scattered by the particle can be expanded in terms of multipoles, of which we find a significant influence of the dipole and quadrupole. The monopolar mode is annihilated as a consequence of the broken symmetry by the incident vortex field [6]. In Fig. 2(c), we plot the different contributions to the torque as a function of $a / \lambda$. The torque arising from the dissipation of the dipole is shown with solid curves and with dashed curves for the quadrupole when only the viscous dissipation in the fluid (blue) or absorption in the particle (black) are considered independently. The total torque is in turn the sum of all four curves. Allowing for a $14 \%$ hydrophone uncertainty on the AV's maximum pressure amplitude $\left(p_{0}=0.8 \pm 0.1 \mathrm{MPa}\right)$, we set bounds for the theoretical evaluation of the total torque with the arising error (gray area). The two torque measurements are also shown (red square and circle for $a=$ 172 and $174 \mu \mathrm{m}$, respectively) with a corresponding $\pm 4 \mu \mathrm{m}$ ( 2 pixels) error in evaluating $a$ and $\pm 1 \mathrm{pNm}$ in evaluating the torque motivated by the observed drag reduction discussed previously. Spanning the range of investigated sizes in experiment would require additional spherical probes with identical absorption that are difficult to obtain commercially. Furthermore, smaller probes will additionally be pushed down the trap by the axial Stokes 
drag $(\propto a)$ until they escape when the negative pulling force fails $\left(\propto a^{3}\right)$. The different axial positions of smaller probes would require to recast the estimation of the torque for the divergence of the incident focused beam.

Our measurements are in good quantitative agreement with our calculations that do not include any adjustable parameters. In the best case (error compensations) the measurement and calculation are in agreement within less than 5\%. However, the error can reach near $100 \%$ (no compensation) as a consequence of the systematic $14 \%$ error in measuring $p_{0}$ that affects, quadratically, the torque and in light of the high contribution of the sphere's visco-elastic absorption (black curves) that rapidly dominates the torque balance and our uncertainty in ascribing the value for the absorption coefficients. Indeed, we did not find additional data in the literature for $\alpha_{\ell}$ and $\alpha_{t}$. Therefore, measuring their value for our spheres could largely improve the torque balance. Importantly, the fact that absorption within the sphere is the dominating driving mechanism contrasts with previous results restricting the description to the viscous boundary layer around the sphere $[23,25]$ or neglecting absorption processes entirely [33]. Finally, within the particle size range considered theoretically, the large contribution of the quadrupolar oscillation mode, even for $a / \lambda<0.05$ [see the close view in Fig. 2(c)], is in contradiction with the common assumption that it can be neglected in the long wavelength (Rayleigh) scattering regime $(a / \lambda \ll 1)$. Though recent analytical calculations under these conditions are recovered here for boundary layer dissipation [49] and particle viscoelastic absorption [40] of the dipolar oscillation (dash-dotted curves in the close view), the dissipation of the quadrupolar mode leads to a torque that rapidly dominates as the radius of the particle increases and must be considered in the full torque evaluation even for small $a / \lambda$ ratios. For example, taking a bond that could generally be considered as lying in the longwavelength regime, $a / \lambda=0.02$, our torque estimation is nearly 10 times larger than the one predicted in that limit when boundary layer dissipation and particle absorption are considered separately (Eq. 13 in Ref. [49] and Eq. (30) in Ref. [40]), mainly as a consequence of the viscous dissipation of the quadrupole vibrating in a viscous fluid (see the Supplemental Material [35] for an extended discussion).

Particle clusters and rotation control.-We additionally observed the rotation of clusters of three or five particles (Fig. 3). The rotation rate of the first is measured to be $32 \mathrm{~Hz}$ and $18 \mathrm{~Hz}$ for the latter. This superior rotation rate compared to the single particle case suggests that the clusters' asymmetry involves an additional mean to transfer OAM. The asymmetry in the scattered field leads to a torque that has been observed in 2D cases for glass fibers [22] or biological cells [25]. We note that the existence of a stable trapping potential for clusters of these dimensions is unexpected from single particle theories of acoustic radiation forces. Accordingly, theses observations call for a deeper understanding of the interaction of $\mathrm{AVs}$ with various particles involving multiple scattering processes and secondary forces and torques [50,51].

Finally, as reported in a preliminary form in Ref. [5] in water and recently for AVs in air [33], it is possible to finetune the rotation rate of the trapped particles by rapidly switching the handedness of the incident AV (Movie 3 in the Supplemental Material [35]). The strategy benefits from the two different time scales involved in the wave phenomena. The fast time scale is determined by the acoustic wave oscillations defined by the acoustic period $T=1 / f=1 \mu \mathrm{s}$. The mean torque arising from the OAM transfer builds up over this time scale. The slow time scale, $\tau$, will ultimately depend on the viscosity of the host fluid and the strength of the driving torque leading to the steady rotation rate. We find that by alternating the wave front handedness at a time scale $T_{0}=400 \mu \mathrm{s}$ (such that $T \ll T_{0}<\tau \sim 1 \mathrm{~ms}$ ), it is possible to fine-tune the rotation rate from the maximum rate available-determined by that obtained at a fixed power

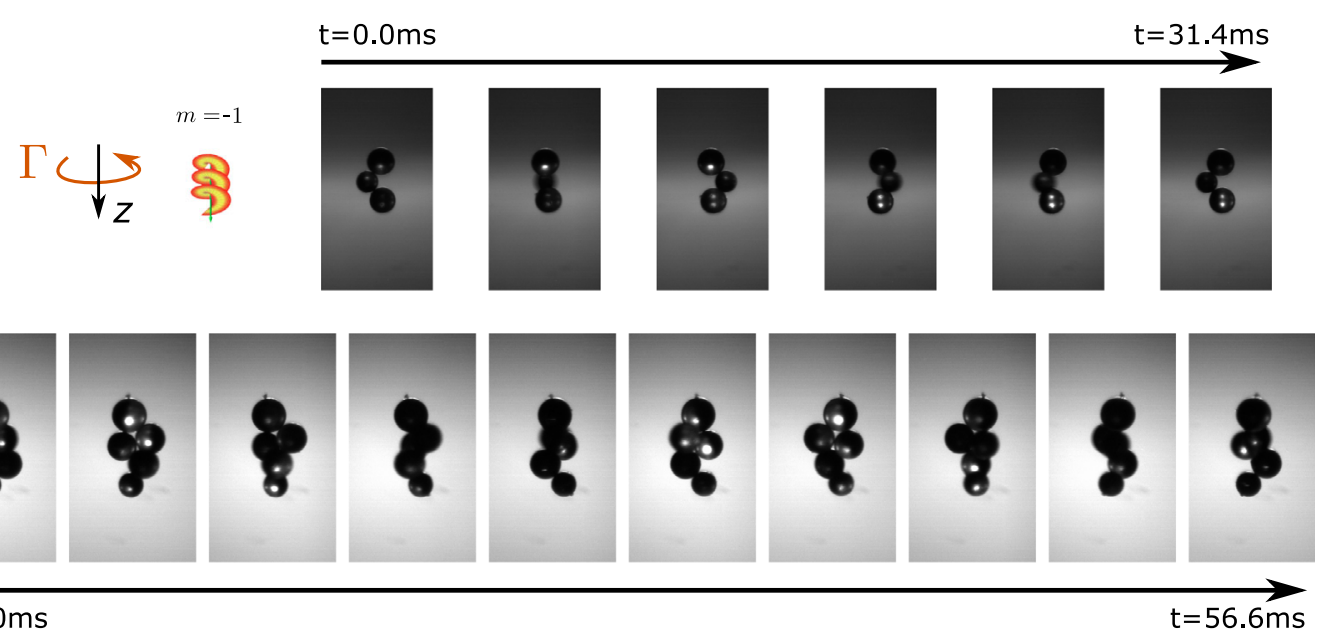

FIG. 3. Simultaneous trapping and rotation of clusters formed by three or five polystyrene particles. The particle sizes are 352, 280, and $320 \mu \mathrm{m}$ (top to bottom) and 380, 320, 320, 320, and $268 \mu \mathrm{m}$ (top to bottom). 
with a single handedness - to nearly zero when during the time duration $T_{0}$, the topological charge is set to $m=1$ half of the time and to $m=-1$ the other half. When the charge is set to be $m=1$ during a period equal to $0.75 T_{0}$, the rotation speed is half of what is obtained with a single handedness during the whole period $T_{0}$. Remarkably, there is no alteration of the trapping position given that the gradient force is invariant with the sign of $m$.

AV-based acoustical tweezers can transfer a controllable amount of OAM to 3D trapped elastic particles. The rotation is driven primarily by the viscous dissipation within a thin viscous boundary layer in the liquid around the particle and the absorption in the particle itself. Both the dipolar and quadropolar vibration modes are dissipated suggesting that the full calculation of the scattered field must include the viscoelasticity of the trapped material and the viscous boundary layer $\delta=(2 \mu / \rho \omega)^{1 / 2}$. Our results suggest that (1) the existence of a theoretical longwavelength regime neglecting the quadrupole can be difficult to translate in experiment; $(2)$ the $(a / \lambda)$ bond for this regime can be material dependant; and finally (3) both absorption in and around the sphere are crucial contributions. While the host medium was here restricted to water, the important role of the rotational flow induced by the viscous attenuation of the AV in the liquid bulk should be considered in evaluating the force and torque in other viscous fluids or at higher frequencies [52-54]. We believe that stable 3D trapping combined with tunable rotation rates has the potential for applications such as contactless microassembly and in situ rheology of small fluid volumes and elastic particles in viscous and complex fluids.

*Present address: Department of Chemical Engineering, Imperial College London.

d.baresch@imperial.ac.uk jean-louis.thomas@upmc.fr

[1] A. Ashkin, J. M. Dziedzic, J. E. Bjorkholm, and S. Chu, Opt. Lett. 11, 288 (1986).

[2] A. Ashkin, J. M. Dziedzic, and T. Yamane, Nature (London) 330, 769 (1987).

[3] K. Svoboda and S. M. Block, Annu. Rev. Biophys. Biomol. Struct. 23, 247 (1994).

[4] D. Baresch, J.-L. Thomas, and R. Marchiano, J. Appl. Phys. 113, 184901 (2013).

[5] D. Baresch, Ph.D. thesis, Université Pierre et Marie Curie-Paris VI, 2014.

[6] D. Baresch, J.-L. Thomas, and R. Marchiano, Phys. Rev. Lett. 116, 024301 (2016).

[7] R. A. Beth, Phys. Rev. 50, 115 (1936).

[8] P. L. Marston and J. H. Crichton, Phys. Rev. A 30, 2508 (1984).

[9] H. He, M. E. J. Friese, N. R. Heckenberg, and H. RubinszteinDunlop, Phys. Rev. Lett. 75, 826 (1995).

[10] N. Simpson, K. Dholakia, and M. Padgett, Opt. Lett. 22, 52 (1997).
[11] M. E. J. Friese, T. A. Nieminen, N. R. Heckenberg, and H. Rubinsztein-Dunlop, Nature (London) 394, 348 (1998).

[12] A. I. Bishop, T. A. Nieminen, N. R. Heckenberg, and H. Rubinsztein-Dunlop, Phys. Rev. Lett. 92, 198104 (2004).

[13] M. McIntyre, J. Fluid Mech. 106, 331 (1981).

[14] L. Rayleigh, The London, Edinburgh, and Dublin Philosophical Magazine and Journal of Science 14, 186 (1882).

[15] G. Maidanik, J. Acoust. Soc. Am. 30, 620 (1958).

[16] T. Wang, H. Kanber, and I. Rudnick, Phys. Rev. Lett. 38, 128 (1977).

[17] F. Busse and T. Wang, J. Acoust. Soc. Am. 69, 1634 (1981).

[18] R. Apfel, J. Acoust. Soc. Am. 70, 636 (1981).

[19] E. H. Trinh, Rev. Sci. Instrum. 56, 2059 (1985).

[20] A. Biswas, E. Leung, and E. Trinh, J. Acoust. Soc. Am. 90, 1502 (1991).

[21] D. Foresti and D. Poulikakos, Phys. Rev. Lett. 112, 024301 (2014).

[22] T. Schwarz, G. Petit-Pierre, and J. Dual, J. Acoust. Soc. Am. 133, 1260 (2013).

[23] A. Lamprecht, T. Schwarz, J. Wang, and J. Dual, J. Acoust. Soc. Am. 138, 23 (2015).

[24] P. Hahn, A. Lamprecht, and J. Dual, Lab Chip 16, 4581 (2016).

[25] I. Bernard, A. A. Doinikov, P. Marmottant, D. Rabaud, C. Poulain, and P. Thibault, Lab Chip 17, 2470 (2017).

[26] K. Volke-Sepúlveda, A. O. Santillán, and R. R. Boullosa, Phys. Rev. Lett. 100, 024302 (2008).

[27] K. Skeldon, C. Wilson, M. Edgar, and M. Padgett, New J. Phys. 10, 013018 (2008).

[28] C. E. M. Demore, Z. Yang, A. Volovick, S. Cochran, M. P. MacDonald, and G. C. Spalding, Phys. Rev. Lett. 108, 194301 (2012).

[29] A. Anhäuser, R. Wunenburger, and E. Brasselet, Phys. Rev. Lett. 109, 034301 (2012).

[30] Z. Hong, J. Zhang, and B. W. Drinkwater, Phys. Rev. Lett. 114, 214301 (2015).

[31] R. Wunenburger, J. I. V. Lozano, and E. Brasselet, New J. Phys. 17, 103022 (2015).

[32] A. Marzo, S. A. Seah, B. W. Drinkwater, D. R. Sahoo, B. Long, and S. Subramanian, Nat. Commun. 6, 8661 (2015).

[33] A. Marzo, M. Caleap, and B. W. Drinkwater, Phys. Rev. Lett. 120, 044301 (2018).

[34] J.-L. Thomas, R. Marchiano, and D. Baresch, J. Quant. Spectrosc. Radiat. Transfer 195, 55 (2017).

[35] See Supplemental Material at http://link.aps.org/ supplemental/10.1103/PhysRevLett.121.074301 for further details on experiments and calculations. The document includes Refs. [4,6,17,20,23-25,36-47].

[36] Y. Takagi, T. Hosokawa, K. Hoshikawa, H. Kobayashi, and Y. Hiki, J. Phys. Soc. Jpn. 76, 024604 (2007).

[37] J. R. Allegra and S. A. Hawley, J. Acoust. Soc. Am. 51, 1545 (1972).

[38] M. Settnes and H. Bruus, Phys. Rev. E 85, 016327 (2012).

[39] M. Wiklund, R. Green, and M. Ohlin, Lab Chip 12, 2438 (2012).

[40] G. T. Silva, J. Acoust. Soc. Am. 136, 2405 (2014).

[41] D. Baresch, J.-L. Thomas, and R. Marchiano, J. Acoust. Soc. Am. 133, 25 (2013).

[42] L. Zhang and P. L. Marston, J. Acoust. Soc. Am. 129, 1679 (2011). 
[43] L. Zhang and P. L. Marston, Phys. Rev. E 84, 065601 (2011).

[44] R. Kono, J. Phys. Soc. Jpn. 15, 718 (1960).

[45] P. S. Epstein and R. R. Carhart, J. Acoust. Soc. Am. 25, 553 (1953).

[46] J. T. Karlsen and H. Bruus, Phys. Rev. E 92, 043010 (2015).

[47] F. Johansson et al., mpmath: A Python library for arbitraryprecision floating-point arithmetic (version 0.18) (2013), http://mpmath.org/.

[48] J.-L. Thomas and R. Marchiano, Phys. Rev. Lett. 91, 244302 (2003).
[49] L. Zhang and P. L. Marston, J. Acoust. Soc. Am. 136, 2917 (2014).

[50] G. T. Silva and H. Bruus, Phys. Rev. E 90, 063007 (2014).

[51] J. Leão-Neto and G. T. Silva, Ultrasonics 71, 1 (2016).

[52] C. Eckart, Phys. Rev. 73, 68 (1948).

[53] A. Riaud, M. Baudoin, J.-L. Thomas, and O. BouMatar, Phys. Rev. E 90, 013008 (2014).

[54] A. Riaud, M. Baudoin, O. BouMatar, L. Becerra, and J.-L. Thomas, Phys. Rev. Applied 7, 024007 (2017). 